\title{
Maternal and gestational influences on childhood blood pressure.
}

\author{
Bonita Falkner
}

Thomas Jefferson University

Follow this and additional works at: https://jdc.jefferson.edu/medfp

Part of the Nephrology Commons, and the Pediatrics Commons Let us know how access to this document benefits you

\section{Recommended Citation}

Falkner, Bonita, "Maternal and gestational influences on childhood blood pressure." (2019).

Department of Medicine Faculty Papers. Paper 252.

https://jdc.jefferson.edu/medfp/252

This Article is brought to you for free and open access by the Jefferson Digital Commons. The Jefferson Digital Commons is a service of Thomas Jefferson University's Center for Teaching and Learning (CTL). The Commons is a showcase for Jefferson books and journals, peer-reviewed scholarly publications, unique historical collections from the University archives, and teaching tools. The Jefferson Digital Commons allows researchers and interested readers anywhere in the world to learn about and keep up to date with Jefferson scholarship. This article has been accepted for inclusion in Department of Medicine Faculty Papers by an authorized administrator of the Jefferson Digital Commons. For more information, please contact: JeffersonDigitalCommons@jefferson.edu. 


\title{
Maternal and Gestational Influences on Childhood Blood Pressure
}

\author{
Bonita Falkner, MD \\ Department of Medicine and Pediatrics \\ Thomas Jefferson University
}

Key words: Childhood, Blood Pressure, Gestational Hypertension, Gestational Diabetes, Preeclampsia, Maternal Diabetes, Maternal Obesity,

Word count: 7,412 including abstract, text, key summary points, questions, and references

Correspondence:

Bonita Falkner, MD

Professor Emeritus

Thomas Jefferson University

833 Chestnut St. Ste. 7000

Philadelphia, PA 19107

Phone: 215-205-2857

FAX: 215-732-6402

Email: bonita.falkner@jefferson.edu 


\section{Abstract:}

Exposures that contribute to a sub-optimal intrauterine environment can have an effect on the developing fetus. Impaired fetal growth that results in low birth weight is an established risk factor for cardio-metabolic disorders later in life. Recent epidemiologic and prospective cohort studies that include the maternal and gestational period have identified maternal and gestational conditions that confer increased risk for subsequent cardio-metabolic disorders in the absence of low birth weight. Maternal pre-conception health status, including chronic obesity and type 2 diabetes increase risk for childhood obesity and obesity related higher blood pressure (BP) in child offspring. Maternal gestational exposures including gestational diabetes, gestational hypertension, and preeclampsia are associated with higher BP in offspring. Other maternal exposures such as cigarette smoke and air pollution also increase risk for higher BP in child offspring. Recent, but limited, data indicate that assisted reproductive technologies can be associated with hypertension in childhood, despite otherwise normal gestation and healthy newborn. Gestational exposures associated with higher BP in childhood can be related to genetics, familial life-style factors, or could also be a consequence of fetal programing. Current and developing research will provide additional insights on gestational exposures and fetal adjustments that increase risk for higher BP levels in childhood. 


\section{Introduction:}

In the prenatal period, the intrauterine environment is critical for optimal fetal growth and organ development. Exposures due to a sub-optimal intrauterine environment can alter the developmental pathway in the fetus in ways that can affect extra-uterine health status later in life. Low birth weight has become an established risk factor for subsequent metabolic and cardiovascular disorders in childhood and in later adulthood. This relationship has been confirmed mainly by large epidemiologic studies on adult populations or cohorts with access to birth records. Experimental studies developed animal models to replicate a compromised intrauterine environment. ${ }^{1,2}$ These models demonstrate that the timing of perturbations in the intrauterine environment have an adverse effect on the fetus that is related to impaired health status in extra-uterine life. Subsequent epidemiologic studies investigated maternal conditions, both prior to conception and perinatal, that could confer a shift in the intrauterine environment to one that is suboptimal for the developing fetus. It is now known that these exposures can have an effect on offspring in childhood and later adulthood that is independent of birth weight. This review will discuss the current state of knowledge on maternal and gestational conditions that influence suboptimal health outcomes in offspring. Other environmental maternal perinatal exposures will be discussed that may have an effect on intrauterine conditions leading to chronic cardiovascular and metabolic health consequences in offspring. This review will focus on maternal and gestational conditions and exposures that appear to have a direct or indirect effect on blood pressure (BP) in childhood. Recent clinical studies on low birth weight that provide insights on the development of hypertension in childhood or later adulthood due to fetal programing will also be reviewed.

\section{Maternal Obesity and Diabetes Mellitus;}


Maternal obesity prior to pregnancy is now recognized as a risk factor for development of obesity in childhood regardless of birth weight. ${ }^{3-6}$ Blood pressure (BP) levels are higher in overweight and obese child offspring of maternal obesity compared to non-obese children. ${ }^{7}$ In a study on mother-offspring pairs from a prospective pregnancy cohort, gestational weight gain (GWG) was monitored throughout pregnancy and offspring were examined at age 9 years. Mothers who exceeded the recommended GWG were more likely to have offspring with greater body mass index (BMI) with greater total fat mass, higher plasma leptin and C-reactive protein, and higher systolic BP. Data from other studies also report higher systolic BP in early childhood among offspring of mothers with greater maternal pre-pregnancy adiposity and greater GWG up to 36 weeks gestation. ${ }^{4,8}$ The higher BP levels among obese offspring are associated with relative insulin resistance and reflect components of metabolic syndrome. Therefore, it is difficult to determine if the abnormal cardio-metabolic outcomes in offspring of maternal obesity are a consequence of fetal programing or are directly related to the childhood obesity resulting from post-natal family environment. ${ }^{9}$ A study by Gaillard et al. ${ }^{10}$ sought to determine if the associations of pre-pregnancy obesity with adverse cardiovascular risk factors in offspring was mediated by heredity, familial lifestyle or intrauterine mechanisms. In a population-based prospective cohort study, the investigators examined mothers, fathers, and their children to investigate associations of both maternal and paternal pre-pregnancy BMI with cardio-metabolic outcomes in children at age 6 years. Higher pre-pregnancy maternal and paternal BMI were associated with higher childhood BMI, total body and abdominal fat mass, systolic BP, insulin levels, and lower high-density lipoprotein cholesterol levels. The associations were stronger for maternal pre-pregnancy BMI than paternal pre-pregnancy BMI. The stronger associations with maternal pre-pregnancy BMI with offspring risk factors suggested the outcomes could be mediated by intrauterine mechanisms. Although maternal obesity is clearly associated with offspring obesity and elevated BP, the evidence for fetal programing of adverse BP and metabolic outcomes in offspring is largely based on experimental models. ${ }^{11}$ Moreover, a recent 
study conducted by the Pregnancy and Childhood Epigenetics (PACE) consortium, examined epigenetic modifications such as DNA methylation in fetal cord blood to determine if there were associations with maternal pre-pregnancy BMI. In this well powered study of over 10,000 mother-newborn pairs, the association of newborn blood DNA methylation with maternal BMI were minimal, indicating that the relationship of maternal obesity with adverse cardio-metabolic risk factors, including elevated BP, in offspring were more likely due to genetic or lifestyle factors. ${ }^{12}$

Insulin resistance is common in both maternal diabetes and maternal obesity and both conditions express risks associated with excess adiposity. However, gestational hyperglycemia can augment the risk for adverse outcomes in offspring. To determine the offspring risk for development of diabetes following intrauterine hyperglycemia, Dabelea et al. ${ }^{13}$ examined young adult sibling pairs in which one was born before the mother developed diabetes and the other sibling was born after the mother had become diabetic and was exposed to hyperglycemia in utero. The risk of diabetes was significantly higher in siblings born after the mother developed diabetes (odds ratio $3.7, \mathrm{P}=0.02$ ). The mean $\mathrm{BMI}$ is also significantly higher among siblings exposed to maternal diabetes compared to siblings born before the mother developed diabetes. ${ }^{14}$ Development of excess adiposity appears to be the seminal risk factor among offspring exposed to maternal hyperglycemia and is detectable in early childhood. ${ }^{15}$ Data from the EPOCH (Exploring Perinatal Outcomes in Children) study examined BMI growth trajectories from birth to age 13 years. BMI growth was not significantly different between exposed and unexposed infants up to 26 months. From 27 months through age 13 years BMI growth was significantly greater among offspring exposed to maternal hyperglycemia compared to unexposed offspring $(P=0.005) .{ }^{16}$ Concurrent with childhood obesity, other related cardiometabolic risk factors are found in offspring of maternal diabetes, including gestational diabetes. ${ }^{17}$ In addition to findings of relative insulin resistance, higher BP at mean age 15 
years, ${ }^{18}$ age 7 years, ${ }^{19}$ and age 3 years ${ }^{20}$ are reported in offspring exposed to intrauterine hyperglycemia compared to unexposed offspring. A meta-analysis of 13 studies on diabetic pregnancy and offspring BP in childhood reported significantly higher systolic BP in offspring of diabetic mothers. The association of higher BP in offspring of diabetic pregnancy was stronger in boys than girls. ${ }^{21}$ Overall, both maternal obesity and maternal diabetes confer heightened risk on offspring for obesity, insulin resistance, and higher BP

\section{Maternal Hypertension:}

Recent reports on associations of maternal hypertension during pregnancy with offspring BP describe a significant positive association. However, in earlier reports, the leading factor in predicting future BP in offspring was low birth weight, ${ }^{22}$ or low birth weight combined with overweight in childhood. ${ }^{23}$ In a large study by Vatten et al. ${ }^{24}$ female adolescent girls, age 13 to 19 years were stratified as offspring of preeclamptic pregnancies or normotensive pregnancies. Offspring of preelamptic pregnancies had significantly higher systolic BP and diastolic BP, and also significantly higher BMI in adolescence. After adjustment for BMI, the differences in systolic and diastolic BP were no longer significant. The authors concluded that the intrauterine exposure to preeclampsia could be causally related to later hypertension but may be confounded by higher BMI during adolescence. A somewhat later meta-analysis of published reports on preeclampsia and offspring BP concluded that nearly all studies demonstrate higher $\mathrm{BP}$ in offspring of women with preeclampsia, in the range of 2 to $3 \mathrm{mmHg}$ systolic pressure. However, the authors noted considerable differences in definition of preeclampsia and potential relevant maternal factors such as age, severity of preeclampsia, maternal BMI, parity, and socio-economic status that should be considered in developing causal relationships between maternal preeclampsia and offspring BP. ${ }^{25}$ 
Subsequent studies examined BP in offspring of mothers with preeclapsia and also offspring of mothers with gestational hypertension. Investigators in the Avon Longitudinal Study of Parents and Children examined data of 6343 offspring participants at age 9 years. Using rigorous definitions of hypertensive disorders of pregnancy, mothers were categorized as normotensive pregnancy $(N=5,345)$, Gestational hypertension $(N=1,118)$, or preeclampsia $(N=205)$. Mothers who had a previous diagnosis of hypertension prior to pregnancy were excluded from analysis. Compared to offspring of normotensive pregnancy, systolic BP was higher in offspring of preeclampsia $(2.05 \mathrm{mmHg}\{95 \% \mathrm{Cl} ; 1.72,3.38\})$ and also in offspring of gestational hypertension $(2.04 \mathrm{mmHg}\{95 \% \mathrm{Cl} ; 1.42,2.67\})$. Diastolic BP was also significantly higher among offspring of preeclampsia $(1.00 \mathrm{mmHg}\{95 \% \mathrm{Cl} ;-0.01,2.10\})$ and offspring of gestational hypertension $(1.07 \mathrm{mmHg}\{95 \% \mathrm{Cl} ; 0.60,1.54\})$. Following adjustment for birth weight and gestational age, the association of preeclampsia with offspring systolic and diastolic BP attenuated and became non-significant. However, these adjustments did not attenuate the association of gestational hypertension with higher offspring BP. ${ }^{26}$ In another report on this cohort, investigators examined offspring of preeclampsia, gestational hypertension, and normotensive pregnancies at offspring age 9 to 12 years. In addition to BP, other measures of vascular function and structure were obtained in the offspring, including brachial artery flow mediated dilation, pulse wave velocity, and brachial artery distensibility. Markers of inflammation, and lipids were also measured. Following adjustments for maternal and offspring BMI, offspring dietary sodium intake, and other potential confounders, significantly higher systolic and diastolic BP was detected in both offspring of preeclampsia and offspring of gestational pregnancy compared to offspring of normotensive pregnancy. No associations were detected among offspring of either hypertensive disorder of pregnancy with the other vascular function measures or with inflammatory markers or lipids. The authors concluded that the association of offspring BP with preeclampsia and with gestational hypertension are consistent with the presence of shared mother-offspring risk factors specific for higher BP, rather than 
additional cardio-metabolic abnormalities specifically due fetal exposure to maternal hypertension. ${ }^{27}$ A portion $(\mathrm{N}=4438)$ of this cohort was re-examined at a mean age of 17 years and included offspring of preeclampsia $(\mathrm{N}=53)$ and gestational hypertension $(\mathrm{N}=431)$ as well as offspring of normotensive pregnancy. In addition to BP and BMI, fasting insulin, glucose, and lipids were measured. The results again demonstrated significantly higher systolic and diastolic BP among offspring of preeclampsia and offspring of gestational hypertension compared to offspring of normotensive pregnancy, following adjustment for potential confounders of age, sex, maternal age at delivery, social-economic class, pre-pregnancy BMI, and smoking in pregnancy. There were no differences in insulin, glucose, or lipids detected between offspring of maternal hypertension and offspring of normotensive mothers. These results suggested that the positive association between maternal hypertensive disorders of pregnancy with offspring BP may be mediated by genetics or familial non-genetic risk factors related to BP. ${ }^{28}$ Moreover, the differences in BP levels between offspring of hypertensive pregnancies and offspring of normotensive pregnancies remain consistent from childhood through adolescence..$^{29}$

Other studies have reported similar findings. Miettole et al. ${ }^{30}$ examined data from the Northern Finland Birth Cohort on offspring at mean age 16 years. They reported higher systolic BP in offspring of gestational hypertension with a tendency for higher cholesterol and apolipoprotein $B$ values compared to offspring of normotensive mothers. Offspring of preeclampsia had higher BP compared to offspring of normotensive mothers; however, this difference was no longer significant following adjustments for confounders. Clinically, preeclampsia is different than gestational hypertension. It remains unclear if the offspring outcomes are also different. A recent systematic review, that included 18 studies, determined that offspring of preeclampsia have significantly higher systolic BP, diastolic BP, and higher BMI compared to offspring of normotensive pregnancies. ${ }^{31}$ In a smaller study, BP characteristics of offspring of mothers with early onset preeclampsia (before 34 weeks gestation) were compared to offspring of mothers 
with late onset preeclampsia. At age 6 to 13 years, systolic BP was significantly higher in offspring of early onset preeclampsia compared to offspring of late onset preeclampsia. ${ }^{32}$

Additional insights on offspring outcomes of hypertensive disorders of pregnancy are conveyed in a report on data from the HUNT study (Nord-Trondelag Health study) in Norway. This cohort includes 15,778 offspring participants at mean age 29 years, and includes 210 sibling groups. BP, anthropometrics, lipids, and C-reactive protein were measured. The offspring exposed to maternal hypertension in pregnancy included 336 exposed to gestational hypertension, 343 exposed to term preeclampsia, and 27 exposed to preterm preeclampsia. Offspring exposed to hypertension in pregnancy had significantly higher levels of systolic and diastolic BP compared to offspring of normotensive pregnancies. It is notable that the difference in BP levels among offspring exposed to hypertensive pregnancy compared to offspring unexposed are at levels quite similar to that described in the childhood reports cited above. Offspring exposed to hypertension in pregnancy also had higher BMI and greater waist circumference than offspring of normotensive pregnancies. The differences in BP and BMI were similar in offspring of gestational hypertension and offspring of term preeclampsia, compared to offspring of normotensive pregnancy. The striking finding in this study was the outcomes among siblings. Siblings who were born to a mother with a normotensive pregnancy had a cardiovascular risk profile, including BP and BMI, that was similar to their sibling who was exposed to a hypertensive pregnancy. These findings indicate that the cardiovascular risk, manifest by higher BP and other BP related risk factors could be attributed to shared genetic factors with the mother and shared environmental factors. ${ }^{33}$ The offspring in the preterm preeclampsia group were all premature, based on gestational age, and had low birth weight. The authors provide little mention of this group other that the sample size was too small to analyze separately. The overall evidence indicates that both maternal gestational hypertension and preeclampsia are 
conditions that increase risk for high BP in offspring, regardless of birth weight, and the risk for elevated BP is heightened with development of excess adiposity in childhood.

\section{Other Exposures During Gestation:}

A maternal behavior that is considered to have an adverse effect on offspring later in life is smoking during pregnancy. An investigation on 8815 adult participants of the 1958 British Birth Cohort detected a link between maternal smoking during pregnancy and offspring adiposity. The investigators measured cardiovascular risk parameters in offspring participants at mean age 45 years. Compared to offspring of non-smokers, offspring of smokers had higher BMI, waist circumference, systolic and diastolic $\mathrm{BP}, \mathrm{HbA} 1 \mathrm{c}$ and triglycerides. Following adjustment for post-natal factors, BP and metabolic risk factors were no longer significant. However, mean $\mathrm{BMI}$ and waist circumference remained significantly higher in offspring of mothers who smoked during pregnancy compared to non-smokers. Mean BMI and waist circumference increased with the number of cigarettes the mother smoked, but were not elevated among offspring of mothers had quit smoking before or early in pregnancy. ${ }^{34}$ Similar data on offspring following prenatal exposure to maternal smoking was also reported in children. Oken et al. ${ }^{35}$ investigated 746 mother-child pairs in Project Viva, a prospective cohort study. Mothers were stratified as never smokers $(69 \%)$, early pregnancy smokers (10\%), or former smokers $(22 \%)$. Child offspring were examined at age 3 years. Compared to offspring of never smokers, offspring of early pregnancy smokers had increased risk for excess adiposity and significantly higher BMI z-score and higher systolic BP $(2.4 \mathrm{~mm} \mathrm{Hg} ; 95 \% \mathrm{Cl},-0.1,4.9)$. Offspring of former smokers were not more overweight than offspring of never smokers, but had higher systolic BP $(1.5 \mathrm{~mm} \mathrm{Hg}$; $95 \%$ $\mathrm{Cl},-0.1,3.2)$. While both offspring of former smokers and offspring of early pregnancy smokers had higher BP compared to offspring of never smokers, offspring of former smokers did not manifest excess adiposity. Whether the BP and BMI patterns remain different in later childhood 
is not known. Oken et al. ${ }^{36}$ also conducted a systematic review and meta-analysis on 14 observational studies on offspring (ages 3-33 years) whose mothers smoked during pregnancy. Overall, child offspring of mothers who smoked during pregnancy were at higher risk for overweight compared to offspring of mothers who did not smoke during pregnancy (pooled adjusted odds ratio 1.50; $95 \% \mathrm{Cl}, 1.36,1.65)$. Effect of prenatal smoking on offspring BP was not addressed in this meta-analysis. A direct effect of maternal smoking during pregnancy on offspring BP has not been confirmed. However, the significant association of maternal smoking during pregnancy with heightened risk for obesity in offspring suggest that offspring could have higher BP related to obesity. Considering the established adverse health effects of cigarette smoke exposure in children and adults, prenatal smoking is strongly discouraged.

In addition to cigarette smoke, there are other inhaled maternal exposures that could have an adverse effect on offspring BP. Particulate matter inhalation from air pollution is reported to be significantly associated with hypertensive disorders of pregnancy, ${ }^{37}$ and preterm birth, ${ }^{38}$ two conditions linked with higher BP in offspring. Reports on air pollution and pregnancy outcomes from Canada describe an association between particulate matter exposure and low birthweight, ${ }^{39}$ and an association of particulate matter with preterm birth that was stronger among women with pre-existing diabetes, asthma, and preeclampsia. ${ }^{40}$ A relationship of maternal exposure to ambient particulate matter during pregnancy and risk for high $\mathrm{BP}$ in childhood was investigated prospectively in a cohort of mothers in the Boston Birth Study ( $N=$ 1293) and their offspring. Offspring were examined between age 3 to 9 years. Analysis of study data identified a marked increase in offspring systolic BP percentile and elevated BP when third trimester particulate matter exposure (PM2.5) was $>13 \mathrm{ug} / \mathrm{m}^{3}$ (highest tertile of PM2.5). ${ }^{41}$ A recent study described a significant association of maternal prenatal air pollution exposure, specifically the particle nitrogen dioxide (NO2), with newborn cord blood DNA methylation. ${ }^{42}$ Another study, conducted on participants in the Children's Health Study ( $N=$ 
459), examined prenatal exposure to NO2 and other particulate matter pollutants, with DNA methylation in cord blood samples of offspring, and childhood BP. The investigators reported that NO2 exposure in the third trimester, compared to other prenatal periods, was associated with greater DNA methylation and higher BP levels in 11 year old children. ${ }^{43}$ ] This study suggested that childhood BP outcomes were related to the gestational timing of fetal exposure to particulate matter in air pollution.

Other chemicals are being investigated for possible relationships with cardio-metabolic risk factors in childhood. Phthalates and bisphenols are environmental chemicals commonly used in consumer and personal care products. These chemicals and their metabolites are considered to be possible endocrine disrupting chemicals. Trasande and Attina ${ }^{44}$ conducted a cross-sectional study on a sub-sample of children who were participants in the National Health and Nutrition Examination Survey 2009-2012 that included an assay of urine samples for metabolites of phthalates. In urine samples from children 6 to 19 years of age, metabolites of low molecular weight phthalates were significantly associated with higher systolic BP z-score in univariate analysis, but the association was no longer significant following multivariable adjustment. A study that measured urinary concentrations of phthalate and bisphenol metabolites in pregnant women found that measureable levels of these metabolites were commonly detected. ${ }^{45}$ Despite several studies on these chemical compounds, some of which describe and adverse effect of prenatal and childhood exposure on child growth and cardiometabolic outcomes, the results of some studies are inconclusive. Further prospective studies are needed to determine if there are quantifiable risks on offspring following prenatal exposure. ${ }^{46}$

Assisted reproductive technologies (ART) may be another type of maternal exposure that could have an effect on offspring BP. ART is a relatively new, but increasingly used, 
strategy in fertility treatment. Little is data are available on long term outcomes of children conceived by ART and result in term pregnancies with apparently healthy children. Scherrer et al. ${ }^{47}$ conducted a study to investigate vascular function on healthy early adolescents (mean age 11.5 years) who were products of a term pregnancy following maternal ART. Children exposed to ART were matched by age, sex, BMI, and social status with children who were term offspring of normal pregnancy. ART cases that involved maternal complications including preeclampsia were excluded. Children conceived by ART had significantly greater endothelial dysfunction (lower brachial artery dispensability), significantly higher PWV, and carotid thickness compared to controls. However, there were no differences in clinic BP levels and elevated BP was not noted. Concurrent experimental studies, by these investigators, on mice found that mice conceived by ART had endothelial dysfunction and increased vascular stiffness with resulting hypertension and premature death. ART mice also had altered methylation of genes involved in vascular nitric oxide production. These experimental findings were consistent with accelerated vascular ageing in products of ART on conception. ${ }^{48}$ In a 4-year follow-up study on the ART conceived healthy adolescents and controls, participants were recalled at age 16 years and 24hour ambulatory BP monitoring was performed. Clinic BP levels were not provided in this report. 24-hour mean systolic and diastolic BP was significantly higher in the ART conceived adolescents compared to control; and $15.4 \%$ of ART conceived adolescents met criteria for hypertension compared to $2.5 \%$ of controls. ${ }^{49}$ Although the data are limited, these findings are sufficiently compelling to add ART to the prenatal history of children with hypertension and without typical risk factors. Further research is needed to determine if hypertension and evidence of cardiovascular dysfunction can be linked with ART.

\section{Low Birth Weight:}


Following the seminal observation by Barker et al. ${ }^{50}$ many epidemiologic studies have demonstrated a link between lower birth weight and adverse cardio-metabolic outcomes in later adulthood. Some of the maternal conditions discussed above, especially preeclampsia, heighten risk for premature delivery as well as lower birth weight for gestational age. Other identified causes of low birth weight include mechanical obstruction of uterine arteries, maternal corticosteroid treatment, and severe protein deficiency. ${ }^{11}$ The recognized adverse outcomes of low birth weight later in life are metabolic and cardiovascular. For example, a systematic review of population studies found that, in most populations, birth weight was inversely related to the risk for type 2 diabetes in adulthood. ${ }^{51}$ Young adults, age 18 to 27 years born preterm with very low birth weight $(<1500 \mathrm{gm})$ have significantly more impaired glucose tolerance and higher BP compared to age/sex matched individuals born at term with normal range birth weight. ${ }^{52}$ Experimental models designed to impair the intrauterine environment, resulting in low birth weight, provide plausible mechanistic pathways indicating that low birth weight is a marker of fetal programing. ${ }^{1,2}$

Prospective childhood studies that include the maternal prenatal period are limited. Reports from the Avon Longitudinal study of parents and Children, discussed above, reported higher BP among offspring of both preeclampsia and gestational hypertension pregnancies compared to offspring of normotensive pregnancies ${ }^{28}$ A secondary analysis from this cohort examined the relative contribution of different growth periods to BP level. At 10 years of age an inverse association of birth weight with systolic BP was detected. In subsequent child growth periods, all growth parameters including weight, height, and weight-for-height were positively associated with systolic BP, indicating that development of excess adiposity during child growth periods was a modifiable determinant of later BP. ${ }^{53}$ In another small but rigorous prospective study, Lurbe et al,,$^{54}$ enrolled healthy full-term infants of uncomplicated pregnancies. The infants were stratified by birth weight as small (SGA), appropriate (AGA) or Large (LGA) for gestational age. 
BP was measured at 2 days of age was positively associated with birth weight. BP and growth parameters were subsequently measured at 6 months, 2 years, and 5 years. At age 5 years, a blood sample was obtained for metabolic parameters including glucose, insulin, and lipids. Each birth weight group gained a similar amount of weight between each exam interval. SGA infants remained the smallest and LGA infants remained the largest at the subsequent exams.

Following 6 months, current weight and weight gain were positively associated with birth weight, and birth weight was not associated with BP level. At age 5 years the birth weight groups were further stratified according to current weight as small average and heavy. Fasting insulin levels were higher in all infants who became heavy at age 5, and were highest among the SGA group. However, an estimate of insulin resistance, using the homeostatic model assessment index (HOMA), was higher in the entire SGA group regardless of weight status at age 5 years. Even the SGA infants who remained small at age 5 years had measures of insulin resistance that were comparable to those who became heavy at age 5 years. The SGA group also had lower high-density lipoprotein-cholesterol (HDL-C) and higher uric acid levels compared to the other birth weight groups suggesting metabolic programing related to low birth weight status. Most participants in this cohort were re-examined at age 10 years. In the growth period from age 5 to age 10 years the impact of weight gain increased and $22 \%$ of children were obese. Children with fasting insulin levels $>15 \mathrm{U} / \mathrm{L}$ had higher office systolic BP, plasma triglyceride and uric acid, and lower HDL-C. No children had hypertension based on 24-hour ambulatory BP monitoring. Maternal obesity increased the risk of becoming obese at age 10 years. These reports track and association of early childhood growth with relative insulin resistance and higher BP by age 10 years. ${ }^{55}$ Barker et al. ${ }^{56}$ linked this childhood growth pattern with subsequent cardiovascular events in an analysis of longitudinal population data from Helsinki, in which a sample of individuals with coronary heart disease as adults were identified. Adults with coronary events had been small at birth and thin at two years of age; but thereafter gained weight rapidly and had insulin resistance later in life. These and other reports indicate that a 
phenotype of lower birth weight paired with developing a high BMI in childhood confers a high risk for high BP in childhood and adverse cardio-metabolic outcomes in later adulthood. ${ }^{57}$

Prematurity, independent of weight for gestational age has been associated with subsequent insulin resistance and hypertension in childhood. ${ }^{1}$ Vohr et al. ${ }^{58}$ examined a sample $(\mathrm{N}=379)$ of extreme preterms, with birth weight less than $1000 \mathrm{~g}$, at age 6 to 7 years of age. Based on clinic BP measurements, $20.6 \%$ had systolic BP $>90^{\text {th }}$ percentile and $10.8 \%$ had systolic hypertension with comparable rates of abnormal diastolic BP. Children with elevated BP or hypertension had higher BMI, triceps skinfold thickness, and waist circumference. Additional analysis found that weight gain velocity from 18 months to school age and maternal gestational diabetes were associated with high BP in early childhood. Among children with $\mathrm{BMI}<85^{\text {th }} \%$, the prevalence of elevated BP was also high with $17 \%$ having high systolic BP and $19 \%$ having high diastolic BP. Among the children with $\mathrm{BMI}<85^{\text {th }} \%$, high $\mathrm{BP}$ was associated with public insurance and maternal gestational diabetes.

Children born preterm have smaller kidneys, relative to body size, due to lower total nephron number. Compared to age-matched normal birth weight children, children born preterm have lower glomerular density and lower glomerular volume, based on renal biopsy samples. ${ }^{59}$ Raaijmakers et al. ${ }^{60}$ conducted a study to determine if renin might modulate the incidence of hypertension associated with prematurity. Prematurely born children with birth weights $<1000 \mathrm{~g}$ $(\mathrm{N}=93)$ were compared to healthy controls $(\mathrm{N}=87)$, at an average age of 11 years. Renal length and renal function, derived from serum cystatin C, were significantly lower in the children born preterm $(\mathrm{P}<0.001)$. Systolic and diastolic BP were significantly higher in the preterm born children $(\mathrm{P}<0.001)$. The odds of having systolic prehypertension among the preterm children was $6.43(2.52-16.4 ; \mathrm{P}<0.001)$ and the odds of systolic hypertension among the preterm was 10.9 (2.46 - 48.4; $\mathrm{P}<0.002)$. Plasma renin activity was $0.54 \mathrm{ng} / \mathrm{ml}$ per hour lower in the preterm 
born children than term born controls $(P=0.001)$. The study findings indicated that hypertension in children born extreme preterm is characterized by low renin hypertension, however the development mechanism for this finding was unknown. In another study that investigated the renin-angiotensin system, South et al. ${ }^{61}$ quantified angiotensin II and angiotensin-(1-7) in the plasma and urine of 175 adolescents born preterm and 51 term born controls. Prematurity was associated with lower plasma angiotensin II and lower angiotensin-(1-7) but overall higher ratio of angiotensin II/angiotensin-(1-7) compared to the term born controls. The difference in the ratio of angiotensin II/angiotensin-(1-7) was greater in females and individuals with overweight/obesity. BP measurements were not discussed in this report although the authors proposed that perinatal renin-angiotensin system programing was more pronounced in females and among those with overweight/obesity which would increase their risk for early cardiovascular disease. Another study examined young adults born preterm (<29 weeks) with age and sex matched controls born at term. Compared to young adults born at term, young adults born preterm had significantly higher BP, smaller kidneys, higher urine albumin/creatinine ratio, and significantly higher circulating angiotensin I levels (36.3; IQR 13.2-62.3 vs 19.4; IQR 9.9-26.1 pg/ml, $\mathrm{P}<001)$. In this study, there were no group differences in renin, Angiotensin II or Ang (1-7). ${ }^{62}$ Despite differences between studies on components of the renin-angiotensinaldosterone system, the very high prevalence of childhood hypertension among children who were extremely premature with low birth weight suggest an underlying alteration in hemodynamic regulation. The observation that the prevalence of high BP is increased in the presence of excess adiposity suggests that excess postnatal growth augments underlying perinatal programing.

\section{Summary:}


Obesity and diabetes are maternal conditions with heightened risk for childhood obesity and higher BP in offspring. During pregnancy, preeclampsia, gestational hypertension and gestational diabetes are associated with higher BP as well as excess adiposity in offspring. There is now evidence that other exposures including maternal cigarette smoking, air pollution, and possible other environment exposures increase the risk for higher BP during childhood among offspring. Although genetic factors and familial lifestyles can mediate associations of maternal and offspring expression of obesity and high BP, maternal exposures, especially prenatal exposures that result in low birth weight and premature birth, may alter the fetal intrauterine environment. The developing fetus can respond to a sub-optimal environment in ways to improve survival, with changes in organ size, neuroendocrine changes, or changes that involve epigenetic modifications. However, the adjustments to survival in-utero may not serve well in the extra-uterine environment and lead to chronic conditions in later life. Epigenetic alterations indicate modifications in DNA function without changes in DNA sequence through DNA methylation, post-translational histone modifications, modifications of nuclear receptors, and microRNAs. ${ }^{63,64}$ Some findings on epigenetic differences associated to prenatal exposures have been reported in adults. However, clinical studies that investigate prenatal epigenetic modification and outcomes in childhood are limited. One such study by Breton et al. ${ }^{65}$ measured DNA methylation of nitric oxide synthase (NOS) and identified an association of percent DNA methylation of NOS1 with carotid intimal media thickness in children. Considering the many potential adverse exposures and the timing of intrauterine exposure, the fetus may experience a treacherous developmental journey to extra-uterine life. Additional research is needed, beginning in childhood, that utilizes molecular strategies to map the pathway from intrauterine exposures and epigenetic modification to evolution of chronic disease markers. For the present time, there are known maternal exposures that increase risk for higher BP in childhood, and many of these exposures are linked with accelerated weight gain and excess adiposity in childhood. In addition to birth weight, maternal health status both prior to pregnancy 
and during pregnancy should be included in assessment of elevated BP in children and adolescents.

\section{Key Summary Points:}

1. Maternal diabetes and obesity are conditions associated with childhood obesity and obesity-associated elevated BP in childhood.

2. Gestational hypertension and preeclampsia are perinatal exposures associated with higher offspring BP in childhood.

3. Extrinsic exposures including maternal cigarette smoking and maternal exposure to air pollution, and assisted reproductive technologies are associated with higher offspring BP in childhood.

4. Causal factors for maternal and gestational influences on childhood BP are genetics, familial life-styles, and fetal programing. 


\section{Multiple choice questions (answers are provided following the reference list).}

1. The most common high risk maternal condition that has been associated with higher BP in child offspring is:
a. Type 2 diabetes
b. Obesity
c. Preeclampsia
d. Air pollution

2. The association of maternal obesity and diabetes with higher BP in child offspring is most likely to be:
a. Directly due to fetal programing
b. Secondary due to development of obesity in childhood
c. Directly due to genetic factors
d. Directly due to maternal age

3. A maternal exposure associated with high BP in childhood that is the result of fetal programing is:
a. Gestational hypertension
b. Maternal obesity
c. Assisted reproductive technology
d. All of above

4. Maternal and gestational influences on higher BP in child offspring can due to:

a. Fetal programing 

b. Genetic factors
c. Familial life-style factors
d. All of above

5. A 10 year old boy is referred for suspected hypertension. His BMI is $>95^{\text {th }} \%$, $\mathrm{BP}$ is $=90^{\text {th }} \%$, physical exam is otherwise normal, On birth history, birth weight was $3.2 \mathrm{Kg}$ and mother has type 2 diabetes before and throughout pregnancy. Additional evaluation should include:
a. 24 hour ambulatory BP monitoring
b. Lipid panel
c. Diet history
d. No further evaluation needed

\section{References:}

1. Gluckman PD, Hanson MA, Cooper C, Thornburg KL. Effect of in utero and early-life conditions on adult health and disease. The New England journal of medicine 2008;359:61-73. 2. Ojeda NB, Grigore D, Alexander BT. Intrauterine growth restriction: fetal programming of hypertension and kidney disease. Advances in chronic kidney disease 2008;15:101-6.

3. Yu Z, Han S, Zhu J, Sun X, Ji C, Guo X. Pre-pregnancy body mass index in relation to infant birth weight and offspring overweight/obesity: a systematic review and meta-analysis. PLoS One 2013;8:e61627.

4. Fraser A, Tilling K, Macdonald-Wallis C, et al. Association of maternal weight gain in pregnancy with offspring obesity and metabolic and vascular traits in childhood. Circulation 2010;121:2557-64. 
5. Kaar JL, Crume T, Brinton JT, Bischoff KJ, McDuffie R, Dabelea D. Maternal obesity, gestational weight gain, and offspring adiposity: the exploring perinatal outcomes among children study. The Journal of pediatrics 2014;165:509-15.

6. Poston L. Maternal obesity, gestational weight gain and diet as determinants of offspring long term health. Best Pract Res Clin Endocrinol Metab 2012;26:627-39.

7. Tan HC, Roberts J, Catov J, Krishnamurthy R, Shypailo R, Bacha F. Mother's prepregnancy BMI is an important determinant of adverse cardiometabolic risk in childhood. Pediatric diabetes 2015;16:419-26.

8. Lawlor DA, Najman JM, Sterne J, Williams GM, Ebrahim S, Davey Smith G. Associations of parental, birth, and early life characteristics with systolic blood pressure at 5 years of age: findings from the Mater-University study of pregnancy and its outcomes. Circulation 2004;110:2417-23.

9. Fleten $\mathrm{C}$, Nystad W, Stigum $\mathrm{H}$, et al. Parent-offspring body mass index associations in the Norwegian Mother and Child Cohort Study: a family-based approach to studying the role of the intrauterine environment in childhood adiposity. American journal of epidemiology 2012;176:83-92.

10. Gaillard R, Steegers EA, Duijts L, et al. Childhood cardiometabolic outcomes of maternal obesity during pregnancy: the Generation R Study. Hypertension 2014;63:683-91.

11. Palinski W. Effect of maternal cardiovascular conditions and risk factors on offspring cardiovascular disease. Circulation 2014;129:2066-77.

12. Sharp GC, Salas LA, Monnereau C, et al. Maternal BMI at the start of pregnancy and offspring epigenome-wide DNA methylation: findings from the pregnancy and childhood epigenetics (PACE) consortium. Hum Mol Genet 2017;26:4067-85.

13. Dabelea D, Hanson RL, Lindsay RS, et al. Intrauterine exposure to diabetes conveys risks for type 2 diabetes and obesity: a study of discordant sibships. Diabetes 2000;49:2208-11. 
14. Lawlor DA, Lichtenstein $\mathrm{P}$, Langstrom N. Association of maternal diabetes mellitus in pregnancy with offspring adiposity into early adulthood: sibling study in a prospective cohort of 280,866 men from 248,293 families. Circulation 2011;123:258-65.

15. Hillier TA, Pedula KL, Schmidt MM, Mullen JA, Charles MA, Pettitt DJ. Childhood obesity and metabolic imprinting: the ongoing effects of maternal hyperglycemia. Diabetes care $2007 ; 30: 2287-92$.

16. Crume TL, Ogden L, Daniels S, Hamman RF, Norris JM, Dabelea D. The impact of in utero exposure to diabetes on childhood body mass index growth trajectories: the EPOCH study. The Journal of pediatrics 2011;158:941-6.

17. Boney CM, Verma A, Tucker R, Vohr BR. Metabolic syndrome in childhood: association with birth weight, maternal obesity, and gestational diabetes mellitus. Pediatrics 2005;115:e2906.

18. Patel S, Fraser A, Davey Smith G, et al. Associations of gestational diabetes, existing diabetes, and glycosuria with offspring obesity and cardiometabolic outcomes. Diabetes care 2012;35:63-71.

19. Tam WH, Ma RCW, Ozaki R, et al. In Utero Exposure to Maternal Hyperglycemia Increases Childhood Cardiometabolic Risk in Offspring. Diabetes care 2017;40:679-86.

20. Wright CS, Rifas-Shiman SL, Rich-Edwards JW, Taveras EM, Gillman MW, Oken E. Intrauterine exposure to gestational diabetes, child adiposity, and blood pressure. American journal of hypertension 2009;22:215-20.

21. Aceti A, Santhakumaran S, Logan KM, et al. The diabetic pregnancy and offspring blood pressure in childhood: a systematic review and meta-analysis. Diabetologia 2012;55:3114-27.

22. Taittonen L, Nuutinen M, Turtinen J, Uhari M. Prenatal and postnatal factors in predicting later blood pressure among children: cardiovascular risk in young Finns. Pediatric research 1996;40:627-32. 
23. Bergel E, Haelterman E, Belizan J, Villar J, Carroli G. Perinatal factors associated with blood pressure during childhood. American journal of epidemiology 2000;151:594-601. 24. Vatten LJ, Romundstad PR, Holmen TL, Hsieh CC, Trichopoulos D, Stuver SO. Intrauterine exposure to preeclampsia and adolescent blood pressure, body size, and age at menarche in female offspring. Obstet Gynecol 2003;101:529-33.

25. Ferreira I, Peeters LL, Stehouwer CD. Preeclampsia and increased blood pressure in the offspring: meta-analysis and critical review of the evidence. Journal of hypertension 2009;27:1955-9.

26. Geelhoed JJ, Fraser A, Tilling K, et al. Preeclampsia and gestational hypertension are associated with childhood blood pressure independently of family adiposity measures: the Avon Longitudinal Study of Parents and Children. Circulation 2010;122:1192-9.

27. Lawlor DA, Macdonald-Wallis C, Fraser A, et al. Cardiovascular biomarkers and vascular function during childhood in the offspring of mothers with hypertensive disorders of pregnancy: findings from the Avon Longitudinal Study of Parents and Children. European heart journal 2012;33:335-45.

28. Fraser A, Nelson SM, Macdonald-Wallis C, Sattar N, Lawlor DA. Hypertensive disorders of pregnancy and cardiometabolic health in adolescent offspring. Hypertension 2013;62:614-20. 29. Staley JR, Bradley J, Silverwood RJ, et al. Associations of blood pressure in pregnancy with offspring blood pressure trajectories during childhood and adolescence: findings from a prospective study. J Am Heart Assoc 2015;4.

30. Miettola S, Hartikainen AL, Vaarasmaki M, et al. Offspring's blood pressure and metabolic phenotype after exposure to gestational hypertension in utero. Eur J Epidemiol 2013;28:87-98.

31. Davis EF, Lazdam M, Lewandowski AJ, et al. Cardiovascular risk factors in children and young adults born to preeclamptic pregnancies: a systematic review. Pediatrics 2012;129:e1552-61. 
32. Lazdam M, de la Horra A, Diesch J, et al. Unique blood pressure characteristics in mother and offspring after early onset preeclampsia. Hypertension 2012;60:1338-45.

33. Alsnes IV, Vatten LJ, Fraser A, et al. Hypertension in Pregnancy and Offspring Cardiovascular Risk in Young Adulthood: Prospective and Sibling Studies in the HUNT Study (Nord-Trondelag Health Study) in Norway. Hypertension 2017;69:591-8.

34. Power C, Atherton K, Thomas C. Maternal smoking in pregnancy, adult adiposity and other risk factors for cardiovascular disease. Atherosclerosis 2010;211:643-8.

35. Oken E, Huh SY, Taveras EM, Rich-Edwards JW, Gillman MW. Associations of maternal prenatal smoking with child adiposity and blood pressure. Obesity research 2005;13:2021-8. 36. Oken E, Levitan EB, Gillman MW. Maternal smoking during pregnancy and child overweight: systematic review and meta-analysis. Int J Obes (Lond) 2008;32:201-10. 37. Xue T, Zhu T, Lin W, Talbott EO. Association Between Hypertensive Disorders in Pregnancy and Particulate Matter in the Contiguous United States, 1999-2004. Hypertension 2018;72:77-84.

38. Trasande L, Malecha P, Attina TM. Particulate Matter Exposure and Preterm Birth: Estimates of U.S. Attributable Burden and Economic Costs. Environ Health Perspect 2016;124:1913-8.

39. Stieb DM, Chen L, Hystad P, et al. A national study of the association between trafficrelated air pollution and adverse pregnancy outcomes in Canada, 1999-2008. Environ Res $2016 ; 148: 513-26$.

40. Lavigne E, Yasseen AS, 3rd, Stieb DM, et al. Ambient air pollution and adverse birth outcomes: Differences by maternal comorbidities. Environ Res 2016;148:457-66.

41. Zhang M, Mueller NT, Wang H, Hong X, Appel LJ, Wang X. Maternal Exposure to Ambient Particulate Matter $</=2.5$ microm During Pregnancy and the Risk for High Blood Pressure in Childhood. Hypertension 2018;72:194-201. 
42. Gruzieva O, Xu CJ, Breton CV, et al. Epigenome-Wide Meta-Analysis of Methylation in Children Related to Prenatal NO2 Air Pollution Exposure. Environ Health Perspect 2017;125:104-10.

43. Breton CV, Yao J, Millstein J, et al. Prenatal Air Pollution Exposures, DNA Methyl Transferase Genotypes, and Associations with Newborn LINE1 and Alu Methylation and Childhood Blood Pressure and Carotid Intima-Media Thickness in the Children's Health Study. Environ Health Perspect 2016;124:1905-12.

44. Trasande L, Attina TM. Association of exposure to di-2-ethylhexylphthalate replacements with increased blood pressure in children and adolescents. Hypertension 2015;66:301-8.

45. Philips EM, Jaddoe VWV, Asimakopoulos AG, et al. Bisphenol and phthalate concentrations and its determinants among pregnant women in a population-based cohort in the Netherlands, 2004-5. Environ Res 2018;161:562-72.

46. Philips EM, Jaddoe VWV, Trasande L. Effects of early exposure to phthalates and bisphenols on cardiometabolic outcomes in pregnancy and childhood. Reprod Toxicol 2017;68:105-18.

47. Scherrer U, Rimoldi SF, Rexhaj E, et al. Systemic and pulmonary vascular dysfunction in children conceived by assisted reproductive technologies. Circulation 2012;125:1890-6.

48. Rexhaj E, Paoloni-Giacobino A, Rimoldi SF, et al. Mice generated by in vitro fertilization exhibit vascular dysfunction and shortened life span. J Clin Invest 2013;123:5052-60.

49. Meister TA, Rimoldi SF, Soria R, et al. Association of Assisted Reproductive Technologies With Arterial Hypertension During Adolescence. Journal of the American College of Cardiology 2018;72:1267-74.

50. Barker DJ, Osmond C, Golding J, Kuh D, Wadsworth ME. Growth in utero, blood pressure in childhood and adult life, and mortality from cardiovascular disease. BMJ (Clinical research ed 1989;298:564-7. 
51. Whincup PH, Kaye SJ, Owen CG, et al. Birth weight and risk of type 2 diabetes: a systematic review. JAMA 2008;300:2886-97.

52. Hovi P, Andersson S, Eriksson JG, et al. Glucose regulation in young adults with very low birth weight. The New England journal of medicine 2007;356:2053-63.

53. Jones $\mathrm{A}$, Charakida M, Falaschetti E, et al. Adipose and height growth through childhood and blood pressure status in a large prospective cohort study. Hypertension 2012;59:919-25.

54. Lurbe E, Garcia-Vicent C, Torro MI, Aguilar F, Redon J. Associations of birth weight and postnatal weight gain with cardiometabolic risk parameters at 5 years of age. Hypertension 2014;63:1326-32.

55. Lurbe E, Aguilar F, Alvarez J, Redon P, Torro MI, Redon J. Determinants of Cardiometabolic Risk Factors in the First Decade of Life: A Longitudinal Study Starting at Birth. Hypertension 2018;71:437-43.

56. Barker DJ, Osmond C, Forsen TJ, Kajantie E, Eriksson JG. Trajectories of growth among children who have coronary events as adults. The New England journal of medicine $2005 ; 353: 1802-9$.

57. Gillman MW. Developmental origins of health and disease. The New England journal of medicine 2005;353:1848-50.

58. Vohr BR, Heyne R, Bann C, et al. High Blood Pressure at Early School Age Among Extreme Preterms. Pediatrics 2018;142.

59. Koike K, Ikezumi Y, Tsuboi N, et al. Glomerular Density and Volume in Renal Biopsy Specimens of Children with Proteinuria Relative to Preterm Birth and Gestational Age. Clinical journal of the American Society of Nephrology : CJASN 2017;12:585-90.

60. Raaijmakers A, Zhang ZY, Claessens J, et al. Does Extremely Low Birth Weight Predispose to Low-Renin Hypertension? Hypertension 2017;69:443-9. 
61. South AM, Nixon PA, Chappell MC, et al. Association between preterm birth and the renin-angiotensin system in adolescence: influence of sex and obesity. Journal of hypertension 2018;36:2092-101.

62. Paquette K, Fernandes RO, Xie LF, et al. Kidney Size, Renal Function, Ang (Angiotensin) Peptides, and Blood Pressure in Young Adults Born Preterm. Hypertension 2018;72:918-28.

63. Vo T HD. Molecular mechanisms underlying the fetal programming of adult disease. J Cell Commun Signal 2012;6:139-53.

64. Bogdarina I WS, King PJ, Burns SP, Clark AJL. Epigenetic modificatiion of the reninangiotensin system in the fetal programmng of hyypertension. Circulation research 2007;100:520-6.

65. Breton CV, Park C, Siegmund K, et al. NOS1 methylation and carotid artery intimamedia thickness in children. Circulation Cardiovascular genetics 2014;7:116-22.

\section{Answers to questions:}

1. $b$

2. $b$

3. $\mathrm{c}$

4. $d$

5. a 